\title{
Optimal operation of regional integrated energy system based on demand-side load response
}

\author{
Peng Fang ${ }^{1}$, Cui $\mathrm{Mao}^{2 *}$,Yuping $\mathrm{Chen}^{2}$, Shan Zhou ${ }^{2}$,Rui You ${ }^{2}$ and Siqi Chen ${ }^{2}$ \\ ${ }^{1}$ Zhejiang Electric Power Industry Association, No. 8, Huanglong Road, Xihu District, Hangzhou City, Zhejiang Province \\ ${ }^{2}$ Zhejiang Huayun Electric Power Engineering Design consulting Co. LTD, No. 1, Huadian Lane, Xiacheng District, Hangzhou City, \\ Zhejiang Province
}

\begin{abstract}
The integrated energy system (IES) has the advantage of improving energy utilization and promoting energy flexibility. From the perspective of demand-side load response, this paper establishes demand-side power, thermal load response, and natural gas demand response models, and then constructs the objective function of the lowest operating cost of the regional IES for combined electric heating and gas supply, using Cplex to perform optimization. Finally, a typical northern park is taken as an example to analyze and verify the feasibility of the model and algorithm. The analysis of the case shows that considering the electric heating gas demand side response will be better than not considering or considering only the single and both responses, not only can reduce operating costs, achieve peak reduction and valley filling, but also reduce abandonment of wind and energy, and increase energy utilization rate.
\end{abstract}

\section{Introduction}

Economic development is driving the growth of energy demand, but the reserves of non-renewable energy such as fossil fuels are insufficient. To improve energy efficiency and ensure the sustainable development of the economy, people proposed IES and introduced the use of renewable energy. IES integrates the power system, thermal system and natural gas system, can realize the comprehensive management and economic dispatch of multiple energy sources, and is an effective way to improve energy utilization. Because of the unstable and intermittent output of renewable energy equipment, how to optimize the operation of IES has become an important issue that needs to be resolved.

To promote the sustainable development of energy, relevant scholars have successively carried out relevant researches on regional integrated energy systems. RIES coordinates and optimizes the distribution, transformation, storage, and consumption of various types of energy such as electricity, heat, and gas, breaking the original independent operation mode, improving system flexibility, making full use of renewable energy, and effectively improving Energy efficiency[1]. Ref.[2] considers economy and environmental protection to establish an optimal dispatching model for the park's integrated energy system with electricity as the core. Ref.[3] is based on a regional grid-connected system based on combined electric and heating dispatch, using Cplex to obtain the most micro-power supply in the dispatch period. Good output and operating costs. Ref.[4] proposed an IES multi-objective optimal dispatch model with electricityto-gas conversion and found that changes in natural gas load affect the economy and pollution emissions of the system by affecting the dispatch of the power system. Ref.[5] proposed a new model of electric-gas IES optimal scheduling considering demand-side load response and dynamic natural gas flow and found that the introduction of response can improve the economics of IES operation. Ref.[6] proposed that the energy storage components in the regional integrated energy system can decouple the thermoelectric connection, thereby reducing operating costs; Ref.[7] combined the greedy mutation strategy to propose an improved CPSO algorithm, which effectively analyzed the operating cost model of the regional integrated energy system ;

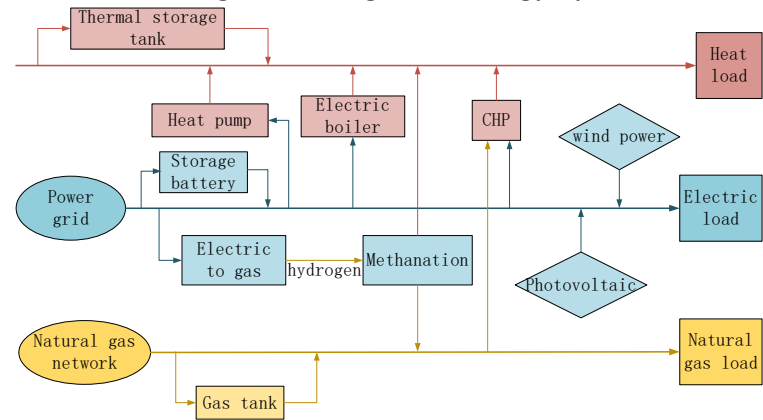

Fig. 1 Regional IES structure

Based on the demand side load response, this paper establishes a demand side electric and thermal load response model, and a natural gas demand response model with the same commodity attributes as electricity. Furthermore, the objective function of the lowest operating cost of the regional IES for the combined supply of electric heating and gas is constructed, and constraints are set for the balance of supply and demand

\footnotetext{
* Corresponding author: 18830251935@163.com
} 
and energy storage equipment, and Cplex is used for optimization.

\section{Electric heating multi-load demand model}

\subsection{Electric load demand side model}

This paper adopts the peak-valley time-of-use price strategy, and divides the 24 hours a day into peak, flat and low periods. This paper uses a widely used electricity price elasticity matrix to solve the power load demand side model.

The total power after the demand side response $q_{z}$ is

$$
q_{z}=q_{t}+\Delta q_{t}=\left[\begin{array}{c}
q_{1} \\
q_{2} \\
\vdots \\
\mathrm{q}_{t}
\end{array}\right]+\left[\begin{array}{llll}
q_{1} & & & \\
& q_{2} & & \\
& & \ddots & \\
& & & \mathrm{q}_{t}
\end{array}\right] E_{d}\left[\begin{array}{c}
\frac{\Delta q_{1}}{q_{1}} \\
\vdots \\
\frac{\Delta q_{t}}{q_{t}}
\end{array}\right]
$$

Where $q_{t}$ is the load power during the $t$ period before the demand side responds; $\Delta q_{t}$ is the change in the load power during the $t$ period after the demand side responds; $E_{d}$ is demand side electricity price elasticity matrix.

\subsection{Thermal load demand side model}

In the $t$ period of this paper, the automatic sliding average model of the heating system temperature can be obtained from the temperature of the water supply $T_{g}(t)$ and the temperature of the return water $T_{h}(t)$ in the heating network, the indoor temperature $T_{n}(t)$ and the outdoor temperature $T_{w}(t)$ in the area.

$\left\{\begin{array}{l}T_{h}(t)=\sum_{j=0}^{J} \alpha_{j} T_{n}(t-j)+\sum_{j=0}^{J} \beta_{j} T_{g}(t-j)+\sum_{j=0}^{J} \gamma_{j} T_{w}(t-j) \\ T_{n}(t)=\sum_{j=0}^{J} \theta_{j} T_{n}(t-j)+\sum_{j=0}^{J} \phi_{j} T_{g}(t-j)+\sum_{j=0}^{J} \omega_{j} T_{w}(t-j)\end{array}\right.$

The best range is set according to the highest $T_{\max }$ and lowest indoor temperature $T_{\min }$.

$$
T_{\min } \leq T_{n}(t) \leq T_{\max }
$$

\subsection{Electric load demand side model}

By analogy with the above-mentioned peak-valley timeof-use electricity price strategy, the peak-valley time-ofuse price natural gas strategy is derived.

The total amount of natural gas after the demand side response $g_{z}$ is obtained:

$$
g_{z}=g_{t}+\Delta g_{t}=\left[\begin{array}{c}
g_{1} \\
g_{2} \\
\vdots \\
g_{t}
\end{array}\right]+\left[\begin{array}{llll}
g_{1} & & & \\
& \mathrm{~g}_{2} & & \\
& & \ddots & \\
& & & \mathrm{g}_{t}
\end{array}\right] E_{q}\left[\begin{array}{c}
\frac{\Delta r_{1}}{r_{1}} \\
\vdots \\
\frac{\Delta r_{t}}{r_{t}}
\end{array}\right]
$$

Where $g_{t}$ is the amount of natural gas loaded during the $t$ period before the demand side responds; $\Delta g_{t}$ is the change in the amount of load electricity during the $t$ period after the demand side responds; $E_{q}$ is demand side natural gas volume natural gas price elasticity matrix.

\section{The optimal operation model}

\subsection{Objective function}

With a dispatch cycle, comprehensive demand response and reasonable arrangement of the contributions of each unit, the total operating cost of the regionally integrated energy system is minimized. The objective function is:

$$
\min F=F_{e}+F_{g}+F_{g e}+F_{w p}+F_{p}
$$

In the formula, $F_{e}, F_{g}, F_{g e}, F_{w p}, F_{p}$ are the power purchase cost from the grid, the natural gas purchase cost, the $\mathrm{P} 2 \mathrm{G}$ raw material cost, the wind and solar abandonment cost, and the maintenance cost of various equipment during the dispatch period.

The specific calculation formula is as follows.

$$
\left\{\begin{array}{l}
F_{e}=\sum_{t=1}^{T}\left(\frac{C_{r s}(t)+C_{r b}(\mathrm{t})}{2} P_{e x}^{t}+\frac{C_{r b}(\mathrm{t})-C_{r s}(t)}{2}\left|P_{e x}^{t}\right|\right) \\
F_{g}=\sum_{t=1}^{T}\left(P_{g}(\mathrm{t}) \mathrm{C}_{C H_{4}}\right) \\
F_{g e}=\sum_{t=1}^{T} C_{C O_{2}} G P_{M, g}(t) \\
F_{w p}=\sum_{t=1}^{T} \alpha\left(\lambda_{w p} P_{w p, f o r e}(\mathrm{t})-P_{w p, r e}(\mathrm{t})\right) \Delta \mathrm{t} \\
F_{p}=\sum_{t=1}^{T}\left(\sum_{i=1}^{N} C_{i}\left|P_{i}(t)\right|\right)
\end{array}\right.
$$

Where $P_{e x}^{t}, C_{r s}(t), C_{r b}(t)$ are the power purchased, the price of electricity purchased and the price of electricity sold in the $t_{\text {period; }} P_{g}(t)$ is the amount of natural gas purchased during the ${ }^{t}$ period; $\mathrm{C}_{\mathrm{CH}_{4}}$ is the unit price of natural gas; $\mathrm{C}_{\mathrm{CO}_{2}}$ is the price of $\mathrm{CO}_{2} ; G$ is the amount of $\mathrm{CO}_{2}$ required to generate unit natural gas; $P_{M, g}(t)$ is the amount of natural gas generated during the $t$ period; $\alpha$ is the cost coefficient of wind abandonment and solar abandonment; $\lambda_{w p}$ is the maximum forecast error coefficient of wind power and photovoltaic output; $P_{w p, f o r e}(t)$ is the output of wind power and 
photovoltaic in the $t$ period Predicted value; $P_{w p, r e}(t)$ is the maximum consumable output of wind power and photovoltaic in the ${ }^{t}$ period; $\Delta t$ is the unit dispatch time; $C_{i}$ is the unit maintenance cost of the equipment $i$; $P_{i}(t)$ is the output of the equipment $i$ in the $t$ period.

\subsection{Constraints}

Supply and demand balance constraints include electrical system balance constraints, thermal system balance constraints, and natural gas balance constraints. The supply-demand balance constraint is expressed as:

$$
\left\{\begin{array}{l}
\min \left[P_{w p, f o r e}(t), P_{w p, r e}(t)\right]+P_{D w}(t)+P_{C H P}(t)+P_{s}(t)=P_{D m d}(t)-P_{c u t}(t) \\
H_{r b}(t)+H_{z r}(t)+H_{C H P}(t)+\mathrm{H}_{P 2 G}(t)+H_{s}(t)=H_{D m d}(t) \\
G_{P 2 G}(t)+G_{g}(t)+G_{s}(t)=G_{D m d}(t)
\end{array}\right.
$$

Where $P_{D w}(t)$ is the electric power obtained from the grid in the $t$ period; $P_{C H P}(t)$ is the amount of electricity generated in the $t$ period of CHP; $P_{s}(t)$ is the output value of the electric energy storage device in the $t$ period; $P_{D m d}(t)$ is the total power of the demand side load in the $t$ period; $P_{c u t}(t)$ is the avoidable load power reduced by the demand side response; $H_{D m d}(t)$ is the total heat load used on the demand side in the $t$ period; $H_{r b}(t), H_{z r}(t), H_{C H P}(t), \mathrm{H}_{P 2 G}(t)$ are the heat generated by heat pumps, electric heating, CHP and P2G equipment in the $t$ period; $H_{s}(t)$ is the output of the thermal energy storage device in the $t$ period; $G_{P 2 G}(t), G_{g}(t), G_{s}(t)$ are the amount of natural gas obtained from the $\mathrm{P} 2 \mathrm{G}$ equipment, natural gas network and gas storage device in the $t$ period, respectively; $G_{D m d}(t)$ is the demand side gas load and the required amount of cogeneration.

The equipment operation constraints is expressed as:

(1) Power supply balance constraint

$$
\begin{aligned}
& \min \left[P_{w p, f o r e}(t), P_{w p, r e}(t)\right]+P_{D w}(t)+P_{C H P}(t)+P_{s}(t)= \\
& P_{D m d}(t)-P_{\text {cut }}(t)
\end{aligned}
$$

Where $P_{D w}(t)$ is the electric power obtained from the grid in the $t$ period; $P_{C H P}(t)$ is the electricity generated in the t-th period of CHP; $P_{s}(t)$ is the output value of the electric energy storage device in the $t$ period; $P_{D m d}(t)$ is the total power of the demand side load in the $t$ period; $P_{c u t}(t)$ is the response of the demand side The reduced avoidable load power.

(2) Heating balance constraint
$H_{r b}(t)+H_{z r}(t)+H_{C H P}(t)+H_{P 2 G}(t)+H_{s}(t)=H_{D m d}(t) \quad$ (9)

Where $H_{r b}(t), H_{z r}(t), H_{C H P}(t), H_{P 2 G}(t)$ are the heat generated by heat pumps, electric heating, CHP and $\mathrm{P} 2 \mathrm{G}$ equipment in the $t$ period; $H_{s}(t)$ is the output of the thermal energy storage device in the $t$ period; $H_{\text {Dmd }}(t)$ is the total heat load used on the demand side in the $t$ period.

(3) Gas supply balance constraint

$$
G_{P 2 G}(t)+G_{g}(t)+G_{s}(t)=G_{D m d}(t)
$$

Where $G_{P 2 G}(t), G_{g}(t), G_{s}(t)$ are the amount of natural gas obtained from the $\mathrm{P} 2 \mathrm{G}$ equipment, natural gas network and gas storage device in the $t$ period; $G_{D m d}(t)$ is the demand side gas load and the required amount of cogeneration.

(4) Coupling equipment output constraint

$$
P_{i, \min } \leq P_{i}(t) \leq P_{i, \text { max }}
$$

Where $P_{i, \min }$ and $P_{i, \max }$ are the minimum and maximum output of each device.

(5) Constraints on energy storage equipment

$$
\begin{gathered}
-C_{i, E S, c} P_{i, \mathrm{cp}} \leq C_{i, \mathrm{ES}}(t) \leq C_{i, E S, \mathrm{f}} P_{i, \mathrm{cp}} \\
\lambda_{\mathrm{min}} P_{i, c p} \leq C_{i, c p}(t) \leq \lambda_{\max } P_{i, c p}
\end{gathered}
$$

Where $C_{i, \mathrm{ES}}(t), C_{i, c p}(t)$ are the power and capacity of the energy storage device in the $t$ period; $C_{i, E S, c}, C_{i, E S, \mathrm{f}}$ are the maximum charge and discharge ratios of the energy storage device $i ; \lambda_{\min }, \lambda_{\max }$ are the maxi-mum and minimum states of charge of the energy sto-rage device $i ; P_{i, c p}$ is the maximum energy storage ca-pacity of the energy storage device $i$.

(6) Constraints on the ramp rate of gas generating units

$$
\left\{\begin{array}{l}
P_{r q}(t)-P_{r q}(t-1) \leq \mathrm{R}_{u p, r q} \\
P_{r q}(t-1)-P_{r q}(t) \leq R_{\text {dow }, r q}
\end{array}\right.
$$

Where $P_{r q}(t), P_{r q}(t-1)$ are the power of the gasfired unit in the $t$ and $t-1$ periods respectively; $\mathrm{R}_{u p, r q}, R_{\text {down, } r q}$ are the limits for the gas-fired unit to increase and decrease the active power in a certain period of time.

(7) Interactive power constraints between IES and electricity/natural gas grid

$$
\begin{aligned}
& P_{e, e x, \text { min }} \leq P_{e, e x}(t) \leq P_{e, e x, \text { max }} \\
& P_{g, e x, \text { min }} \leq P_{g, e x}(t) \leq P_{g, e x, \text { max }}
\end{aligned}
$$

Where $P_{e, e x}(t), P_{g, e x}(t)$ are the power interaction values with the power grid and natural gas grid in the $t$ period; $P_{e, e x, \min }, P_{e, e x, \max }$ are the upper and lower limits of power interaction with the grid; $P_{g, e x, \max }, P_{g, e x, \min }$ are the upper and lower limits of power interaction with the natural gas grid of $100 \mathrm{~m}^{3}$ and $-100 \mathrm{~m}^{3}$, respectively. 


\section{Case analysis}

\subsection{Background}

This article takes a typical regional IES in northern my country as an example. The system includes the WT, PV, CHP, HP, electric boilers, P2G and energy storage devices. Taking 24 hours a day as the scheduling duration, the unit scheduling time is 1 hour. The demand side elasticity coefficient of electricity price is -0.2 , and the cross elasticity coefficient is 0.03 ; the demand side elasticity coefficient of time-of-use natural gas is -0.58 , and the cross elasticity coefficient is 0.15 . The best indoor temperature is $24^{\circ} \mathrm{C}$, the highest temperature is $28^{\circ} \mathrm{C}$, and the lowest temperature is $18^{\circ} \mathrm{C}$.

To verify that IES considers the advantages of demand-side load in this article, several other demandside methods are listed for comparison.

Method 1: Demand side response is not considered

Method 2: Only consider the demand side power response

Method 3: Only consider demand side natural gas response

Method 4: Consider the demand-side power and thermal response

Method 5: Consider the response of demand side power, heat and natural gas

\subsection{Optimization results}

For the demand-side response not considered, only the demand-side power response is considered, only the demand-side natural gas response is considered, the demand-side power and thermal response are considered, and the demand-side power, thermal, and natural gas responses are compared.

Regardless of which load response on the demand side is studied, the peak-to-valley difference of method 5 is significantly smaller than the other four methods. That is, when considering the IES demand-side power, thermal and natural gas load response ratios, the single and the second load response ratio is not considered, and the peak and valley are reduced. The difference is the advantage of peak shaving and valley filling.

It can be seen from Figure 2 to Figure 4 that no matter which load response on the demand side is studied, the peak-to-valley difference of method 5 is significantly smaller than the other four methods, that is, when considering the load response ratio of IES demand side power, heat and natural gas, or considering the single and the other, it has the advantage of reducing the peak-valley difference and achieving peak-shaving and valley-filling.

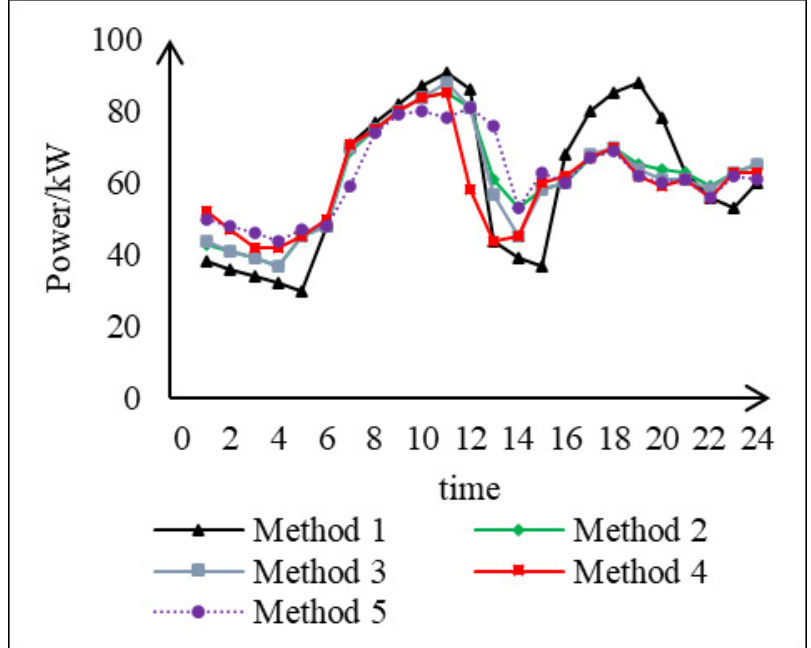

Fig.2 Optimization curve of demand side power load in different modes

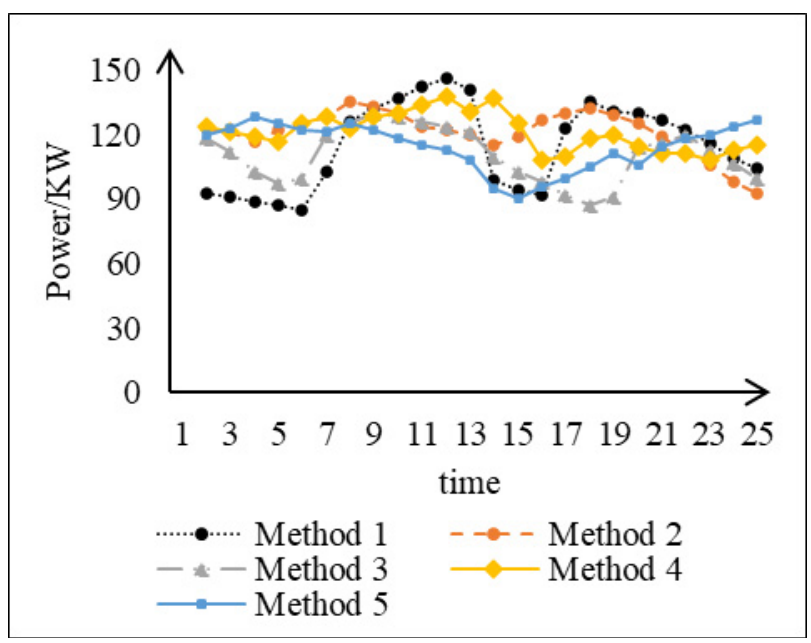

Fig. 3 Optimization curve of heat load on demand side in different modes

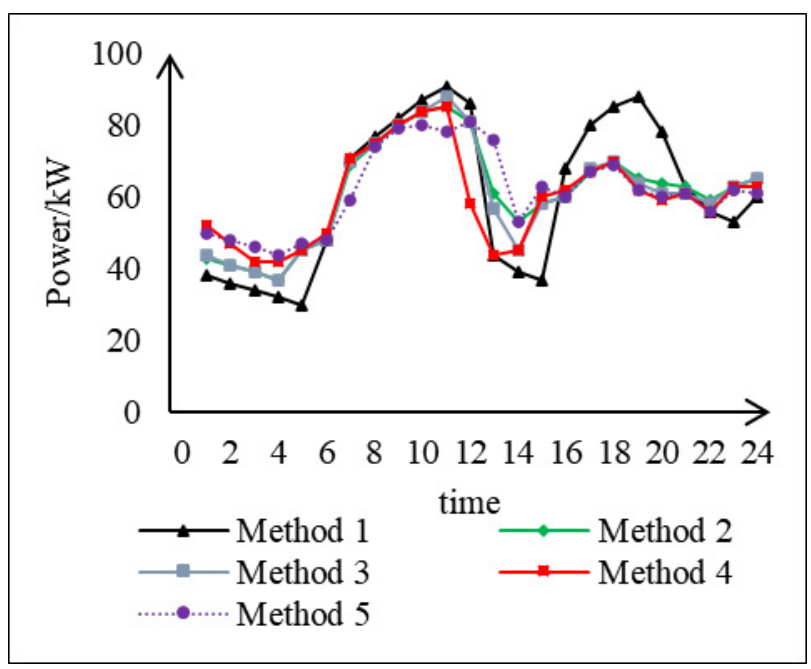

Fig. 4 Optimization curve of demand side natural-gas load in different modes

From Table 1, under the condition of multi-energy fusion, the energy of electric heating and gas is converted to each other, which promotes the increase of energy utilization. Establish a cost-minimum objective 
function that takes into account the cost of abandonment of wind and abandonment of light, taking into account the ability to absorb wind and solar. When the wind and wind output is high, increase the cost coefficient of abandoning wind and light, so that the system can absorb more wind and wind output.

Table 1. System energy utilization efficiency, wind

abandonment and light rejection rate in different modes

\begin{tabular}{|c|c|c|c|}
\hline $\begin{array}{c}\text { Met } \\
\text { hod }\end{array}$ & $\begin{array}{c}\text { Energy } \\
\text { utilization } \\
\text { rate/\% }\end{array}$ & $\begin{array}{c}\text { Wind } \\
\text { curtailment } \\
\text { rate/\% }\end{array}$ & $\begin{array}{c}\text { Light } \\
\text { curtailment } \\
\text { rate/\% }\end{array}$ \\
\hline 1 & 81.3 & 12 & 8 \\
\hline 2 & 86.78 & 10.2 & 7.2 \\
\hline 3 & 86.2 & 11.1 & 7.6 \\
\hline 4 & 89.56 & 9.2 & 6.6 \\
\hline 5 & 91.32 & 7.4 & 5.4 \\
\hline
\end{tabular}

In summary, considering the demand-side electric, heating, and gas load response in the regionally integrated energy system can significantly reduce the peak-to-valley difference in energy consumption on the demand side, improve the energy utilization efficiency in the system, and reduce the amount of wind and solar abandonment and system operating costs.

\section{Conclusion}

This paper establishes an RIES operation model of electric heating and gas demand-side response. Taking a typical park as an example, through different scenarios, the conclusion is drawn: considering the demand-side response to the system dispatchability, the introduction of electricity, heat, especially natural gas demand The side model, through the mutual coupling of the electric heating gas network, plays an important role in peak shaving and valley filling and energy utilization. Based on the peak-valley time-of-use price strategy of electricity, the peak-valley time-of-use price method of natural gas is analogized, and the role of natural gas's time-of-use price is also considered in the system to establish a gas load model. At the same time, the demand side peak-valley time-of-use price strategy is used to coordinate the energy flow in the system to achieve the goal of saving operating costs.

\section{References}

1. Zhang Wei, Dong Xinxin, Sun Weiqing, etc. Research on Integrated Energy System in Energy Internet [J]. Automation Instrumentation, 2017, 38(1): 12-15.

2. Yu Bo, Wu Liang, Lu Xin, et al. Optimal dispatch method of regional integrated energy system[J]. Electric Power Construction, 2016,37(1):70-76.

3. LI Zhengmao, ZHANG Feng, LIANG Jun, et al. Optimization on microgrid with combined heat and power system[J]. Proceedings of the CSEE, 2015, 35(14): 3569-3576.

4. ZENG Hong, LIU Tianqi, HE Chuan, et al. Multiobjective optimization for integrated natural-gas and electricity energy system considering power-togas $[\mathrm{J}]$. Electrical Measurement \& Instrumentation, 2019, 56(8): 99-107.

5. ZHANG Yining, HE Yubin, YAN Mingyu, et al. Optimal dispatch of integrated electricity-natural gas system considering demand response and dynamic natural gas flow $[\mathrm{J}]$. Automation of Electric Power Systems, 2018, 42(20): 1-10.

6. Dichen Liu, Hengrui Ma, Bo Wang, et al. Operation optimization of regional integrated energy system with combined cooling, heating and power and energy storage $[\mathrm{J}]$. Electricity system automation, 2018, 42(4): 113-120,141.

7. Liu Hong, Chen Xingyi, Li Jifeng, etc. Economic dispatch of regional electric heating integrated energy system based on improved CPSO algorithm [J]. Electric power automation equipment, 2017, 37(6): 193-200. 